J3eA, Journal sur l'enseignement des sciences et technologies de l'information et des systèmes,

Volume 3, Hors-Série 1, 6 (2004)

DOI : http://dx.doi.org/10.1051/bib-j3ea:2004606

(C) EDP Sciences, 2004

Reconnaissance de feux piétons en envionnement urbain avec un assistant personnel

D. Moreno Eddowes et J. Lopez Krahe

Laboratoire GRAII, A-145

Université Paris 8

F-93526 Saint-Denis, France

http://ufr6.univ-paris8.fr/desshandi/

daniel.moreno@campus.uab.es, jlk@ai.univ-paris8.fr 


\title{
Reconnaissance de Feux Piétons en Environnement Urbain avec un Assistant Personnel
}

\author{
Daniel Moreno Eddowes, Jaime Lopez Krahe \\ Labo GRAII, A-145, \\ Université Paris 8, 93526 St. Denis \\ http://ufr6.univ-paris8.fr/desshandi/ \\ daniel.moreno@campus.uab.es, jlk@ai.univ-paris8.fr
}

Résumé - Nous présentons un projet pour la détection des Feux Piétons pour personnes handicapées visuels. Cette application est implémentée sur un système standard mobile et autonome, un agenda électronique (PDA) avec une caméra vidéo incorporée. L'interprétation est basée sur l'analyse des scènes prises par la camera vidéo.

Ce système complet intègre aussi d'autres fonctionnalités adaptées aux personnes aveugles, répertoire, gestion de rendez-vous, par reconnaissance des empreintes vocales.

Le traitement est divisé en deux étapes distinctes : l'étape de segmentation et l'étape de reconnaissance. Dans la première étape, l'image est traitée pour trouver les contours des objets d'intérêt. Dans la deuxième étape, on utilise des méthodes structurales sur les formes sélectionnées afin de décider si le Feux piéton est Vert (silhouette en mouvement) ou Rouge (silhouette statique).

Cet équipement doit nous permettre à moyen terme l'apprentissage et la reconnaissance de multiples objets pour se convertir en une aide technique adaptable.

Mots clés : Segmentation d'Images, reconnaissance des formes, aides techniques, distance d'édition, aides compensatrices aux handicaps.

Nous remercions «Pocket Entreprises » pour les facilités qui nous ont été accordées pour l'utilisation du matériel.

\section{INTRODUCTION}

L'objectif du projet est d'obtenir une meilleure qualité de vie pour les personnes handicapés visuels, pour acquérir une certain autonomie.

L'utilisation d'un PDA (assistant personnel) est une bonne solution étant donné que c'est un Système portable, autonome et générique avec un prix accessible.

Actuellement les PDA sont suffisamment puissants et peuvent faire une quantité élevée de calculs (processeur à $400 \mathrm{Mhz}$ [1]). L'évolution est rapide et constante. A moyen terme on trouvera des PDA avec des caractéristiques similaires aux ordinateurs actuels.

Cette application est insérée dans un projet de développement d'aide technique adaptable pour les personnes handicapées, et totalement implémenté sur un PDA. Il s'agit d'un matériel standard qui peut évoluer avec les développements logiciels.

\section{PRESENTATION DU PROBLEME}

Le problème de la reconnaissance à priori est difficile, par la complexité de la scène : le changement de position des feux dans l'image, les différentes tailles qu'ils peuvent avoir de par la distance de la saisie ou de l'optique, les différentes formes des silhouettes, les grandes variations de leurs couleurs (Fig.1, Fig.2) et les conditions d'éclairage. Quelques cas posent des problèmes théoriques (ou heuristiques) intéressants : passages en deux temps ou informations contradictoires (Fig. 7).

Une base de données avec 200 images est disponible librement sur http://ufr6.univ-paris8.fr/desshandi// de manière à permettre d'autres résultats comparatifs.

Nous avons séparé les images en deux types, en fonction des conditions d'éclairage pendant le jour et pendant la nuit. Les images prises pendant la journée ont une grande variation d'intensité sur les plans RGB, et cela n'est pas un critère déterminant. Par ailleurs, dans les images prises pendant la nuit, la couleur peut être utilisée comme un facteur conducteur afin de pouvoir discriminer les régions non utiles (Fig.1).

En fonction de ces critères, nous pouvons effectuer deux implémentations différentes dans l'étape de segmentation.

La deuxième étape reçoit une image binaire segmentée. Cette étape sera la même pour les images pendant la journée et les images de la nuit.

L'objectif est de garantir à $100 \%$ un taux de reconnaissance des feux verts de manière à assurer la sécurité de la traversée. Ceci implique une augmentation des taux de la classe de rejet. Notons que les fausses alarmes sont nombreuses, et qu'un critère unique basé exclusivement dans la couleur ne peut pas être suffisant.

\section{IMPLEMENTATION DU SYSTEME}

Nous présentons une approximation des méthodes utilisées dans chacune des deux étapes, l'étape de Segmentation et l'étape de Reconnaissance. 


\section{A. Étape de Segmentation}

L'étape de Segmentation détermine les contours des possibles formes que vont remplir les critères de détection exigées. La Segmentation est divisée en deux processus :

1- Elimination des parties de l'image sans intérêt: en utilisant un seuil pour la couleur, et aussi pour la localisation dans l'image, pour le cas des images prises pendant la nuit. Pendant la journée on utilise d'autres critères de connaissance qui peuvent nous permettre de chercher la localisation des feux dans la scène, comme la recherche des bandes du passage clouté ou le mât de feux (Fig. 2).

2- Détection des contours par morphologie mathématique [2] dans les images prises pendant la nuit., et avec l'utilisation du gradient [3],[7] dans les images de la journée.

Une fois que nous avons trouvé les contours, on passe à l'étape de reconnaissance.

Dans la (Fig. 3) on peut observer les résultats de la segmentation à partir des images, et aussi les contours transformés en courbe discrète fermée.

\section{B. Étape de Reconnaissance}

Dans cette étape nous partons de l'image du contour binaire transformé en courbe discrète fermée et simple 4connexe.

La première approximation est d'utiliser le codage de Freeman [4] pour coder des modèles (Fig. 4). Ensuite on code toutes les formes que l'on trouve dans l'image segmentée. En deuxième lieu, avec les chaînes obtenues pour le codage, on trouve la chaîne la plus semblable en utilisant la distance d'édition [4], [6] et un codage structural des critères multiples de détection, on détermine avec un seuil si c'est possible de décider si on a trouvé une silhouette de feux ou non.

\section{RESULTATS}

Cette approche s'applique bien au problème de reconnaissance des feux. On a partagé la solution en deux, et les résultats sont différents.

L'étape de Segmentation rencontre les problèmes du traitement d'images. Il y a beaucoup d'approximations pour essayer de résoudre la segmentation, surtout pour les images prises pendant la journée où la diversité des images est très grande, on ne peut pas trouver une méthode optimale. Avec notre implémentation, en utilisant les propriétés de la scène, on a un fonctionnement satisfaisant.

On a fait la classification en trois groupes: feux rouge, feux vert, non classé. Avec le test on a obtenu les suivants résultats :

\begin{tabular}{|c|c|c|c|}
\hline & Rouge & Vert & Non classé \\
\hline Rouge simple-J & 70 & 5 & 25 \\
\hline Vert simple-J & 3 & 64 & 33 \\
\hline Rouge double-J & 60 & 5 & 35 \\
\hline Vert double-J & 3 & 59 & 38 \\
\hline Rouge simple-N & 90 & 1 & 9 \\
\hline Vert simple-N & 2 & 92 & 6 \\
\hline Rouge double-N & 75 & 10 & 15 \\
\hline Vert double-N & 8 & 77 & 15 \\
\hline
\end{tabular}

$\mathrm{J}$ - pendant le jour $\mathrm{N}$ - pendant la nuit

\section{CONCLUSIONS}

La solution apportée pour le problème est bien définie. Par ailleurs il y a des problèmes dans la méthode de Reconnaissance, à cause d'autres facteurs non dépendants du système. La technologie utilisée, par exemple, peut en effet causer des problèmes. La caméra vidéo utilisée, ne permet pas de faire un zoom optique pour prendre une meilleure image de la région d'intérêt. On trouve donc un problème de résolution dans la reconnaissance des petits feux dans la scène (Fig.5).

On trouve d'autres problèmes comme celui présente dans la (Fig.6). Dans cette image, on observe que nous nous trouvons dans le cas où il y a 2 feux, avec un fonctionnement indépendant. On peut se trouver dans le cas qu'un objet (voiture, personne, etc.) couvrant le feu le plus proche, mais on peut regarder l'autre. Si le feu le plus proche est rouge, et l'autre vert, le système peut reconnaître le feu vert, et pas le feu caché.

Finalement on peut observer dans la (fig.7) un cas pathologique. Il y a deux feux, un à côte de l'autre dans un carrefour. Celui de droite correspond au passage de la rue perpendiculaire, il ne faut pas passer quand il es vert !

\section{REFERENCES}

[1] PDAs http://www.lepdashop.com/

[2] Jean Serra. Image analysis and mathematical morphology. Academic Press, London. 1982.

[3] John C. Russ. The image processing handbook. 2a edition. CRC Press, 1995.

[4] L. Miclet. Méthodes structurelles pour la reconnaissance des formes. Éditions Eyrolles, Cnet-Enst 1984.

[5] J. M . Chassery, A. Montanvert Géométrie discrète. Hermes, Paris, 1991

[6] A. Cornuéjos, L. Miclet, Apprentissage artificiel. Eyrolles, Paris, 2002

[7] H. Maitre, Le traitement des images. Hermes, Paris, 2003. 

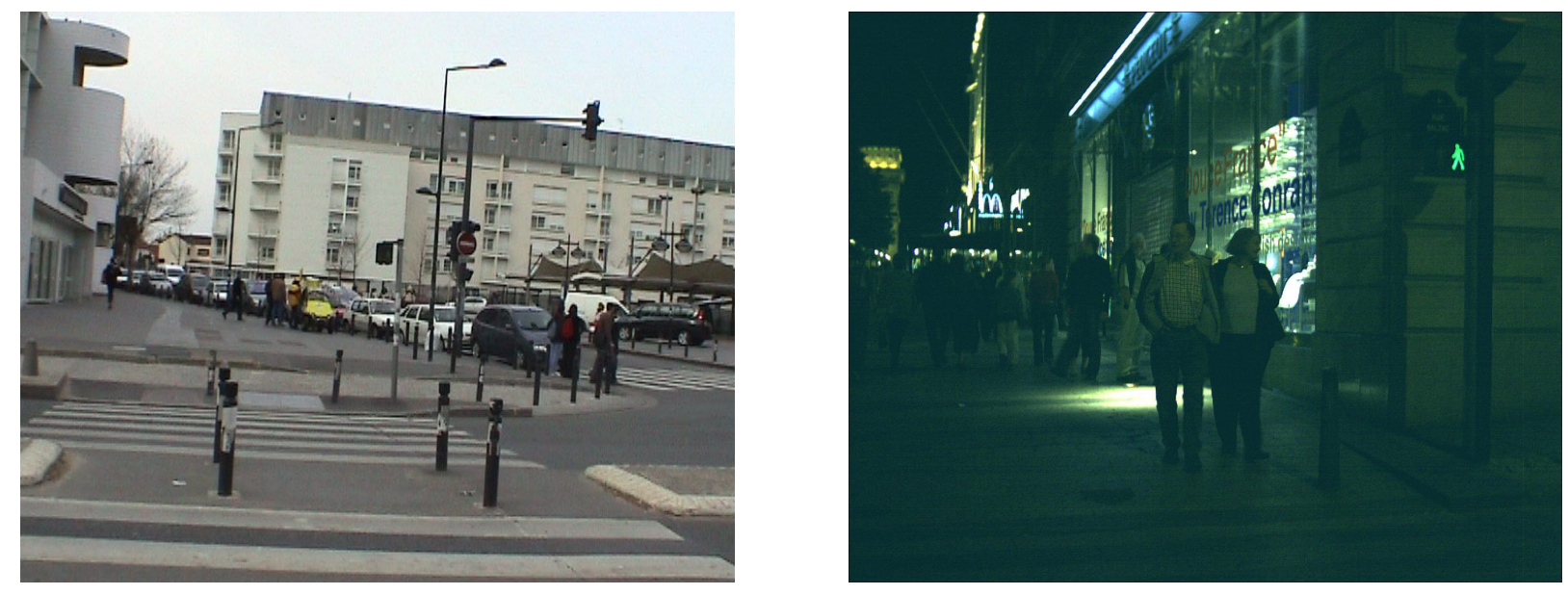

Fig. 1. Différence entre les images (Jour, Nuit).
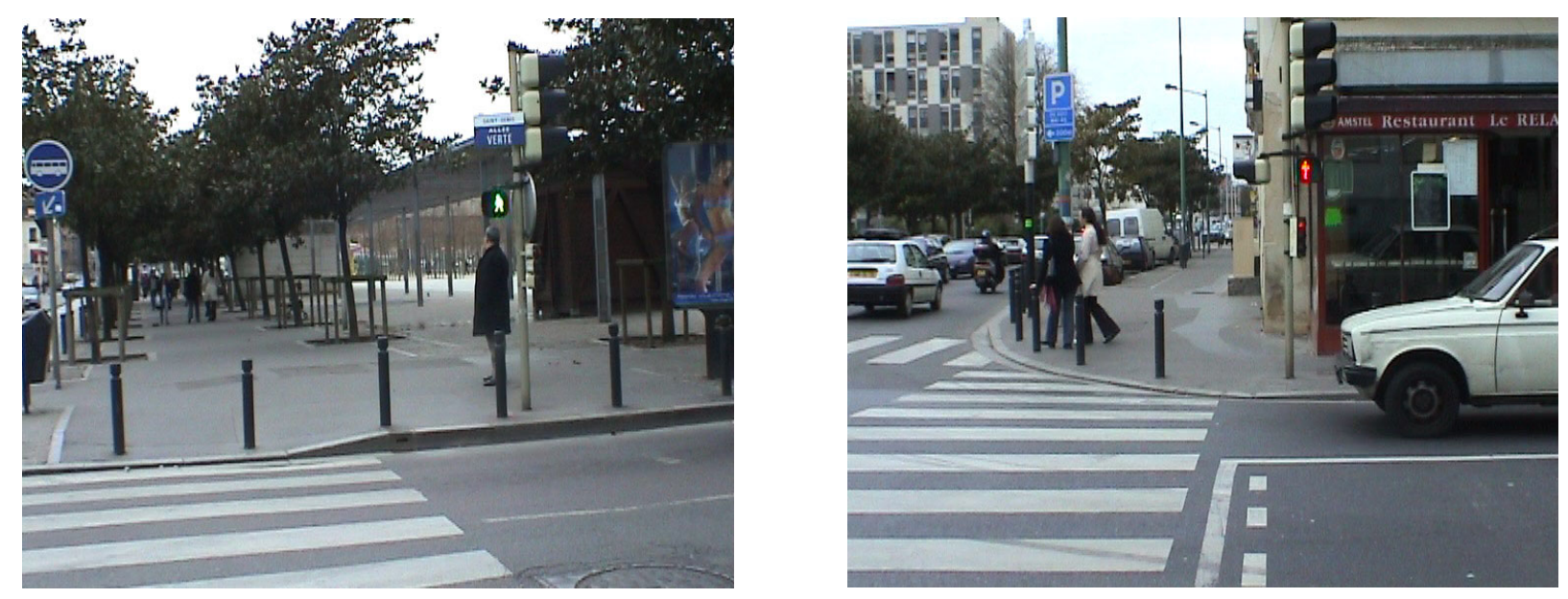

Fig. 2. Situation du passage de piétons et le mât de feux.
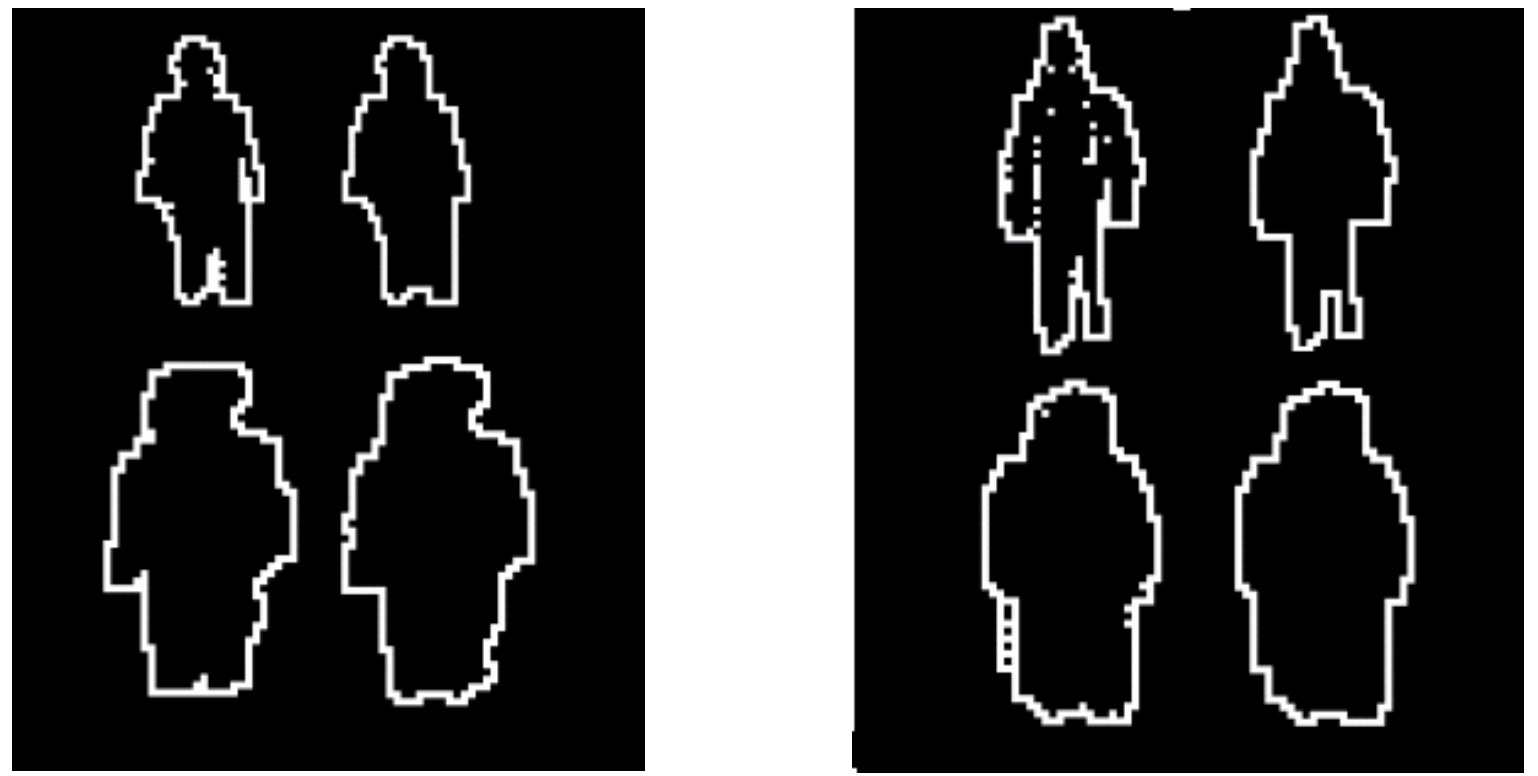

Fig. 3.a Résultats après la segmentation des feux rouges et transformé en courbe discrète fermée. 

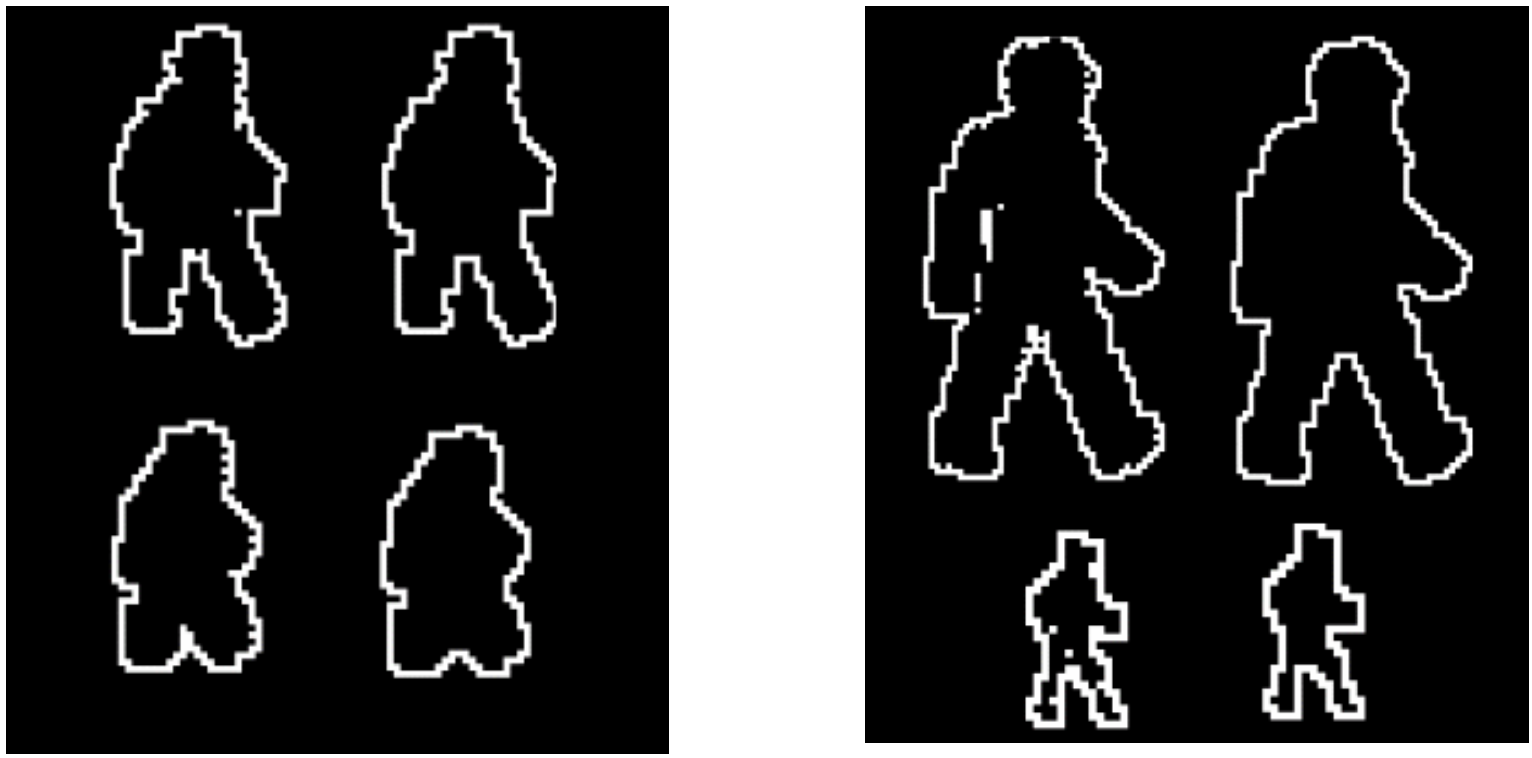

Fig. 3.b Résultats après la segmentation des feux verts et transformé en courbe discrète fermée.

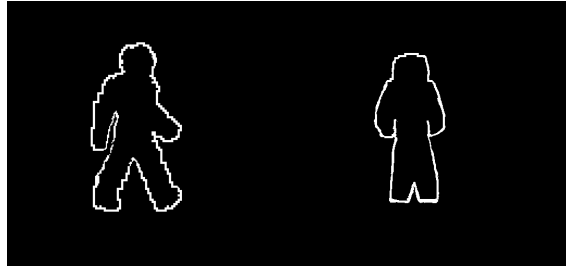

Fig. 4 Échantillon des modèles utilisés.
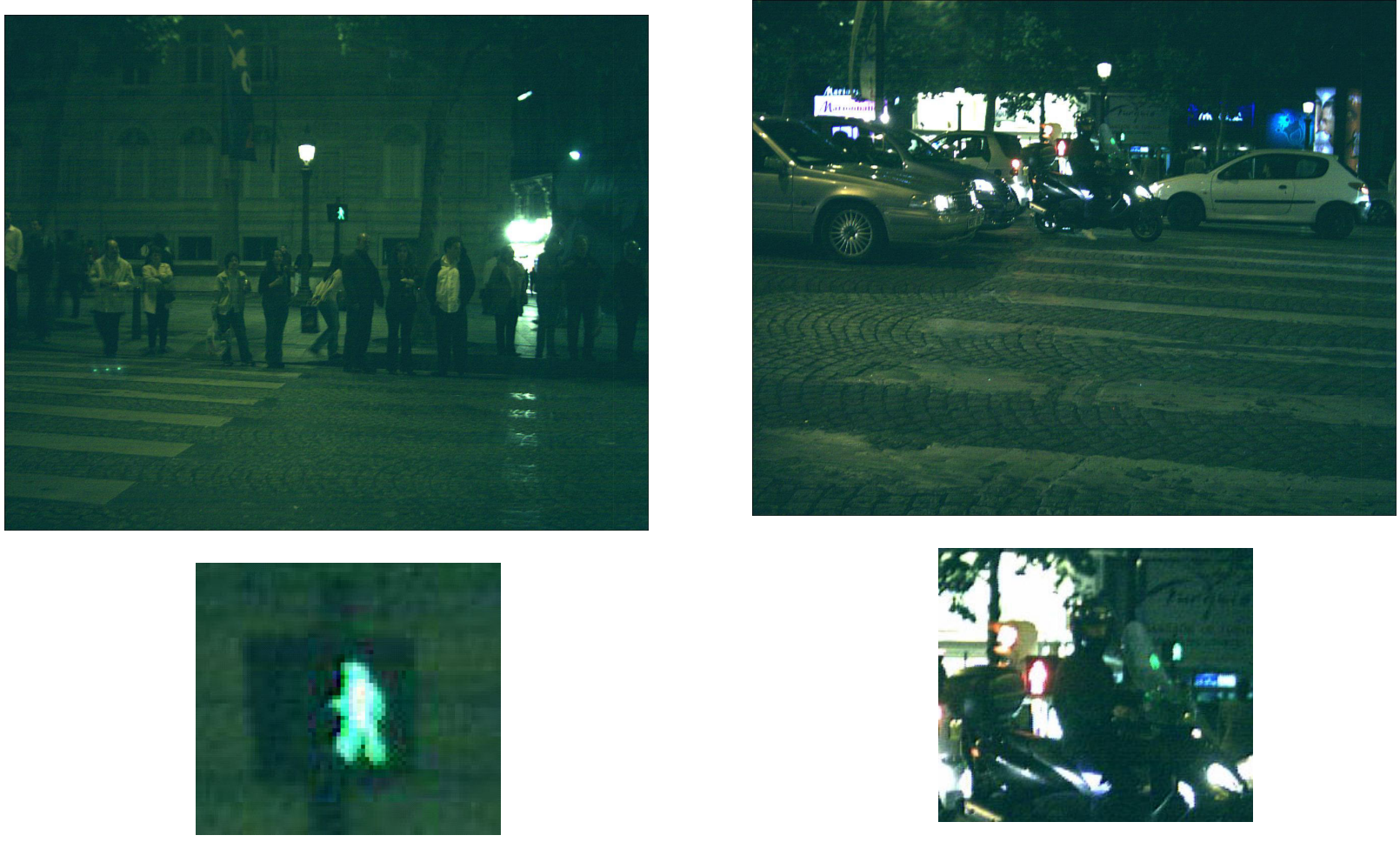

Fig. 5 Feux distante et l'ampliation.

Fig. 6. Deux feux dans la même image. 


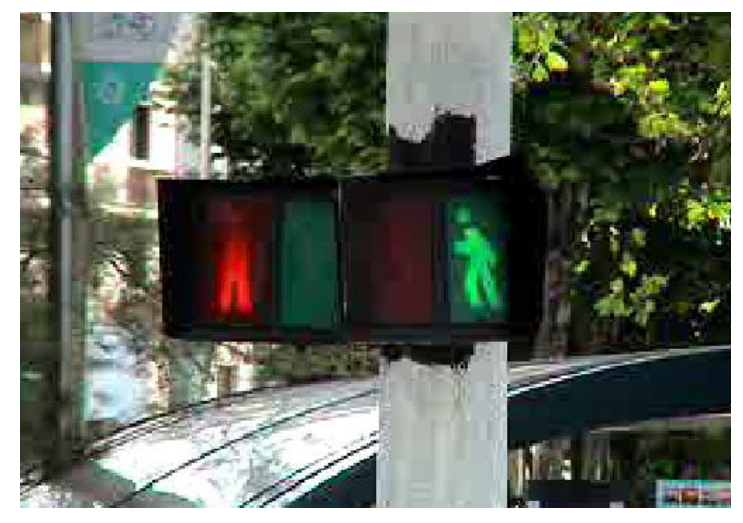

Fig. 7. Cas pathologique. 